Research Article

\title{
Estimation of Arc Welding Pressure Pipeline Weld Peaking Parameters Based on Data Prediction
}

\author{
Pu Liao $\mathbb{D}^{1},{ }^{1}$ Guixiong Liu $\mathbb{D}^{1},{ }^{1}$ and Ningxiang Yang $\mathbb{D}^{2}$ \\ ${ }^{1}$ School of Mechanical and Automotive Engineering, South China University of Technology, Guangzhou 510640, China \\ ${ }^{2}$ Guangdong Province Special Equipment Testing and Research Institute Zhuhai Testing Institute, Zhuhai 519640, China \\ Correspondence should be addressed to Guixiong Liu; megxliu@scut.edu.cn
}

Received 24 August 2021; Accepted 8 November 2021; Published 23 December 2021

Academic Editor: A. M. Bastos Pereira

Copyright $\odot 2021 \mathrm{Pu}$ Liao et al. This is an open access article distributed under the Creative Commons Attribution License, which permits unrestricted use, distribution, and reproduction in any medium, provided the original work is properly cited.

Peaking parameter is the key content in the regular inspection of the pressure pipeline. Solving the problem of the peaking measurement method defined by a standard cannot be applied to a situation in which there exists a weld surface with reinforcement and misalignment. In this paper, a peaking estimation method based on data prediction was proposed to estimate the contour information of the base metal at the weld joint using the contour point set data of the base metal part of the weld. Herein, the longitudinal weld peaking estimation method based on a piecewise logistic regression (PLR) and the girth weld peaking estimation method based on a piecewise Bayesian linear regression (PBLR) were studied, and the midpoint of the two symmetrical points of the base metal on either side of the weld was used as a reference for calculating the peaking. Finally, we collected the surface profile data of longitudinal weld pressure pipes with diameters of $155 \mathrm{~mm}, 255 \mathrm{~mm}, 550 \mathrm{~mm}$, and $600 \mathrm{~mm}$ and the surface profile data of girth weld pressure pipes with diameters of $120 \mathrm{~mm}, 130 \mathrm{~mm}, 140 \mathrm{~mm}$, and $170 \mathrm{~mm}$. This weld seam data used the data estimation method proposed in this article and traditional long short-term memory and fitting methods. The results showed that the proposed data prediction method could accurately predict the position of the base metal, and the theoretical mean absolute error of the peaking estimation based on the PBLR and PLR could attain $0.06 \mathrm{~mm}$ and $0.07 \mathrm{~mm}$, respectively, which meets the parameter measurement requirements of related verification fields.

\section{Introduction}

The manufacturing specification of a welded pressure pipe has a clear stipulation on peaking. Peaking affects the appearance and shape of the pipe, produces stress concentration, and reduces the bearing capacity of the components. In a high-temperature environment, large peaking leads to cracks in the pipe, which may cause pressure pipe cracking $[1,2]$. The peaking of the welding seam refers to the deviation of the butt welding seam of the revolving shell relative to the tangent of the warp or weft, that is, the height of the convex or concave part, and it needs to use customized template and ruler to complete the measurement. Figure 1 shows the standard definition of the longitudinal and girth weld peaking measurement methods [3].

The related detection industry has been using the lowefficiency manual detection method for a long time. Thus, designing a universal inspection method in the surface feature inspection of different welds is a hot research topic. At present, scholars in related fields mainly used the peaking detection method based on ultrasonics. For example, in literature [4], two ultrasonic probes were symmetrically placed on either side of the weld and close to the inner surface of the pipe. The value was read at the joint by the ultrasonic probe with a known qualified peaking, compared with the cylinder with an excessive peaking to detect whether the peaking of the cylinder was qualified. In literature [5], a special shape template was designed, and the relationship between the template and the distance between the two sides of the weld base metal was measured via an ultrasonic probe to estimate the peaking parameters. When facing the peaking parameters of different sizes of pressure pipes, the template needs to be redesigned and processed. Moreover, the applicable range is less. Generally, the peaking of the 
pressure pipe occurs when the plate is pressed into a cylinder. In this case, the peaking measurement is relatively simple. After welding, the joint is filled with solder making it impossible to obtain an accurate measurement. However, in the process of using the pressure pipe, the peaking changes $[6,7]$. Therefore, this paper studies a method of peaking measurement that can be used for postwelding.

The inspection model that uses structured light to image the weld surface has been adopted by several scholars. In literature [8], optical and field emission scanning electron microscopy was used on the cross-sectional view of hardened depth cases. In literature [9], an automatic pipe size measurement system was developed. The system uses the measurement data from 72 measurement laser scanners and sensors and can detect 19 types of pipe size parameters through the trigonometric functional relationship between the measurement parameters. In literature [10], the laser scanning sensor is used to generate the surface point cloud data in the welding process, and the deformation of the welding parts in the welding process is detected according to the point cloud data. Literature [11] used a structured light detection model to generate the weld bead surface profile. After the image is processed via a noise reduction technique, the weld bead shape can be quickly extracted. A structured light surface imaging method can better reflect weld bead surface profile than an ultrasonic detection method; furthermore, the model is simpler. In recent years, more companies have launched corresponding structured light sensor products, such as KEYENCE, SmartRay, and CONNEX, whose sensor surface measurement accuracy has reached the um level, which could better be applied to the field of weld surface detection. For example, in literature [12], the above sensors were used to obtain weld pool surface profile data, and literature [13] used a three-dimensional structured light sensor to image the weld seam and designed a point cloud data processing method to realize weld seam tracking.

To measure the angular parameters of the pressure pipe after welding, the contour information of the base material of the pressure pipe covered by the welding material must be obtained. This kind of problem falls under sequence data predictions $[14,15]$. In literature [16], a multiobjective optimization based on a genetic algorithm was applied to determine the laser welding optimal design input parameters. Some common data prediction methods applied to weld contours include curve fitting and long short-term memory (LSTM). The curve fitting method considered the point cloud of the base metal part of the pressure pipeline to fit a specific shape curve or polynomial and considered the predicted coordinates of the fitting results in the welding part as the welding seam parameter detection index. For example, literature [17] used a third-order polynomial to fit the surface contour point set. However, this method was only suitable for simple weld feature classification. Literature [18] reconstructed the spatially complex curved joint model using the cubic smoothing spline algorithm and detected the characteristic parameters of the lap joint, which could be applied to welding tracking effectively. The LSTM data prediction method was also applied in the field of welding owing to its excellent complex timedomain and spatial-domain signal relevance. For example, literature $[19,20]$ designed an LSTM network to predict the trend of a molten pool in the welding process. In literature [21], LSTM was used to train the data of a weld surface in the welding process to predict the welding quality and the quality level of weld surface defects. However, the LSTM-based prediction method needs online training and predictions; moreover, the algorithm is time-consuming.

The main contributions of this paper are as follows:

(1) We analyze why the welding seam peaking measurement method defined by the standard cannot be applied to the actual pressure pipeline inspection.

(2) We propose the longitudinal weld peaking estimation method based on a piecewise logistic regression (PLR) and the girth weld peaking estimation method based on a piecewise Bayesian linear regression (PBLR).

(3) We design a peaking measurement prototype based on a laser profile sensor. The PLR and PBLR method estimation effects were better than the traditional data predicting algorithm.

The paper is organized as follows. In Section 2, the design details of a weld surface device based on a laser profile sensor are outlined. In Section 3, an overview of the peaking estimation algorithms is detailed. Various experimental results are provided to confirm the validity of the proposed methods in Section 4. Finally, conclusions are provided in Section 5.

\section{Weld Peaking Measuring Device Based on Laser Profile Sensor}

Figure 2 shows a device for measuring weld peaking based on a structured light model comprising a laser profile sensor, mobile station, and computer. The laser profile sensor is the LJ-V7080 laser profile sensor of KEYENCE, the focal length of its built-in camera is $32 \mathrm{~mm}$, and the wavelength of the built-in laser is $405 \mathrm{~nm}$. The measuring ranges of the sensor in the $x$ - and $y$-axis directions are $20 \mathrm{~mm}$ and $46 \mathrm{~mm}$, respectively. The sensor is installed directly above the cylinder to be measured, and the imaging distance of the sensor is within the range. The linear laser emitted by the sensor hits the weld surface of the pressure pipeline to be measured. The camera inside the sensor captures the image near the linear laser range in real time, and the point cloud data are output to the computer via the internal algorithm. The sensor is fixed on the bracket so that the line laser is parallel to the cross section of the pressure pipe cylinder; thus, the sensor can move in the $z$-axis direction. During the detection process, the pressure pipe cylinder and the laser sensor must be kept tangent to the moving direction. 


\section{Modeling and Numerical Analyses}

Based on the point set of the surface base metal output from the laser profile, this paper analyzed the measurement method of the peaking from the longitudinal weld and the girth weld.

\subsection{Estimation Method of Longitudinal Weld Peaking of} Pressure Pipeline Based on Piecewise Logistic Regression. Figure 3 shows an estimation method of peaking for longitudinal weld surface with reinforcement based on a standard peaking measurement method. In the standard, the distance between the reinforcement point of the base material protrusion at the joint and the design arc (coiled steel plate design) in the $y$-axis direction is the parameter of the peaking value. The reinforcement point of the protrusion at the joint position is covered by the welding material, and its exact position is not known; therefore, we need to estimate the coordinates of the contact points of the joints on both sides based on the point cloud data near the weld. Here, we estimated the extension of the base metal profile on both sides near the weld in the welding area and used the distance between the midpoint $y_{\text {pre }}$ of the two symmetrical points at the junction of the base metal and the welding material and the design arc as the peaking $\delta$. Under normal circumstances, the actual steel plate rolling cylinder joint at both ends may appear owing to misalignment, that is, there is a defect misalignment. Figure 4 shows the proposed peaking estimation method for a weld surface with reinforcement and misalignment. Due to the misalignment, the left and right side joints no longer overlap. In this case, it is necessary to calculate the distances $\delta_{1}$ and $\delta_{2}$ between the midpoints $y_{\text {pre }}^{\text {left }}$ and $y_{\text {pre }}^{\text {right }}$ of the two symmetrical points in the $x$-axis direction at the junction of the base metal and the welding material and the design arc, respectively, and consider the maximum of the two as the peaking. Therefore, predicting the coordinates of the contact points of the joints on either side of the weld is the key research content of this measurement index.

The common logistic regression model is as follows:

$$
f(x)=\frac{1}{1+e^{-x}} .
$$

When the total amount of the model is within $(0,1)$, the prediction data attain saturation, and the prediction effect is poor for the growth trend data. Based on this model, the growth rate $k$, total amount $T$, and bias $b$ are introduced, and the logistic regression model is modified as follows:

$$
f(x)=\frac{T}{1+e^{-k(x-b)}} .
$$

At different nodes, the growth rate $k$ and the total amount $T$ might change with different nodes. To clarify the changes of the growth rate $k(x)$ and growth amount $T(x)$, the change point $C P=\left\{c p_{1}, c p_{2} \ldots c p_{m}\right\}$ is introduced into the sequence $S=\left\{s_{1}, s_{2} \ldots s_{n}\right\}$ of length $n$, and the growth rate adjustment $\lambda=\left\{\lambda_{1}, \lambda_{2} \ldots \lambda_{m}\right\}$ is defined. Then, the growth rate at any position of sequence $S$ is the base ratio $k$ plus the growth rate adjustment $\lambda_{i}$ of this point, i.e., $k+a_{i} \lambda_{i}, i=1, \cdots, m$, where $a_{i}$ is given as

$$
a_{i}= \begin{cases}1, & x \geq c p_{i} \\ 0, & \text { else. }\end{cases}
$$

The offset $b$ in different change points can be adjusted accordingly; the adjusted partial mass $v_{i}$ of the change point $c p_{i}$ is as follows:

$$
v_{i}=\left(c p_{i}-b-\sum_{j<i} v_{i}\right)\left(1-\frac{k+\sum_{j<i} \lambda_{i}}{k+\sum_{j \leq i} \lambda_{i}}\right) .
$$

The modified logistic regression model is as follows:

$$
f(x)=\frac{T(x)}{1+\exp \left\{-\left[k+a^{T} \lambda\right]\left[x-b-a^{T} \nu\right)\right\}} .
$$

The logistic regression model is a special case of the generalized logistic regression curve, which is an $S$-shaped curve and has strong expansibility. Based on the model, curve trend parameters were added $\beta$. In different change point areas, the parameter $\beta$ can be changed with the change point $\beta$ adjusted as follows:

$$
\beta_{i}=\beta+a_{i} \lambda_{i}, \quad i=1, \cdots, m
$$

The improved logistic regression model, that is, the piecewise logistic regression (PLR) model proposed in this paper, can be expressed as follows:

$$
f_{i}(x)=\frac{T(x)}{\left.1+\left(\beta_{i}+a_{i}^{T} \lambda\right) \exp \left\{-\left[k+a_{i}^{T} \lambda\right]\left[x-b-a_{i}^{T} \nu\right)\right]\right\}}
$$

After the regression calculation of the model parameters $\lambda, k, b$, and $\beta$, the follow-up data can be predicted according to $(7)$.

\subsection{Estimation Method of Girth Weld Peaking of Pressure} Pipeline Based on Piecewise Bayesian Linear Regression. Figure 5 shows the method proposed in this paper for estimating the peaking of the girth weld surface with reinforcement. Similar to the estimation of the longitudinal weld peaking, peaking was determined as the linear distance between the estimated coordinate $y_{\text {pre }}$ and the base metal at the joint $\delta$, and the linear equation of the base metal was determined by the point cloud fitting line $f_{\text {fit }}(x)$ in the base metal region. Similar to the longitudinal weld, Figure 6 shows the method for estimating the peaking of the weld surface with excess reinforcement and misalignment. In this case, it was necessary to calculate the distance between the estimated coordinates $y_{\text {pre }}^{\text {left }}$ and $y_{\text {pre }}^{\text {right }}$ at the joint and the base metal line on both sides and consider the maximum value as peaking.

In the method of coordinate estimation at the contact point of the two sides of the girth weld, the piecewise Bayesian linear regression (PBLR) model is used to predict the joint. 


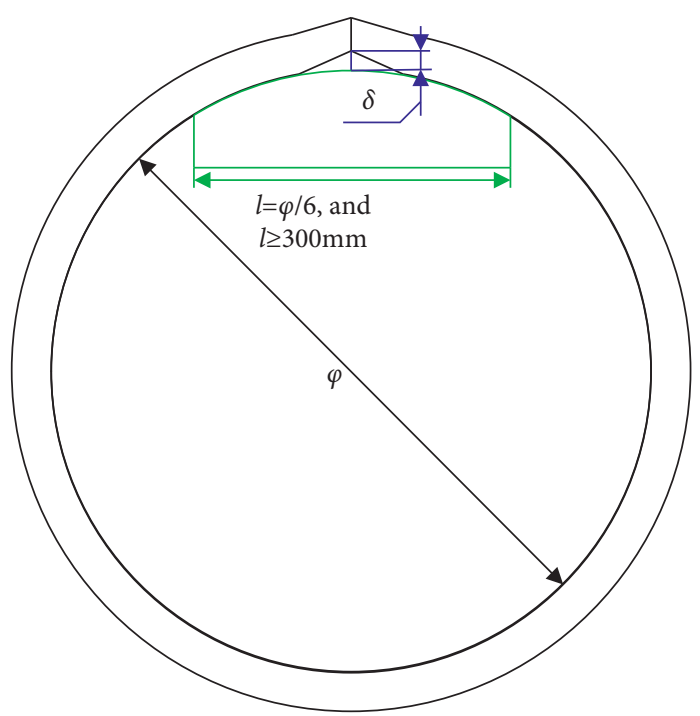

(a)

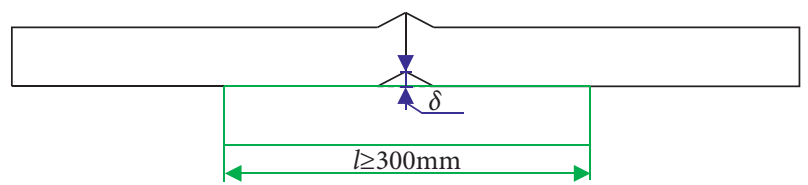

(b)

Figure 1: Standard definition of (a) longitudinal weld joint peaking and (b) girth weld joint peaking measurement methods.

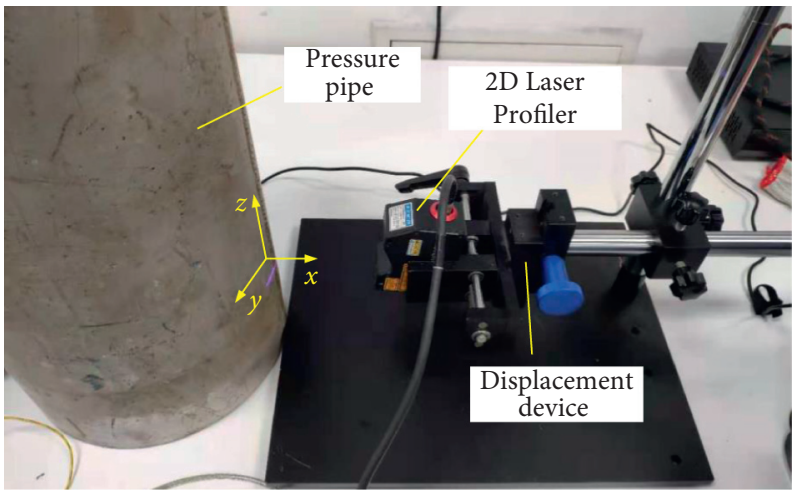

FIGURE 2: Weld peaking measuring device.

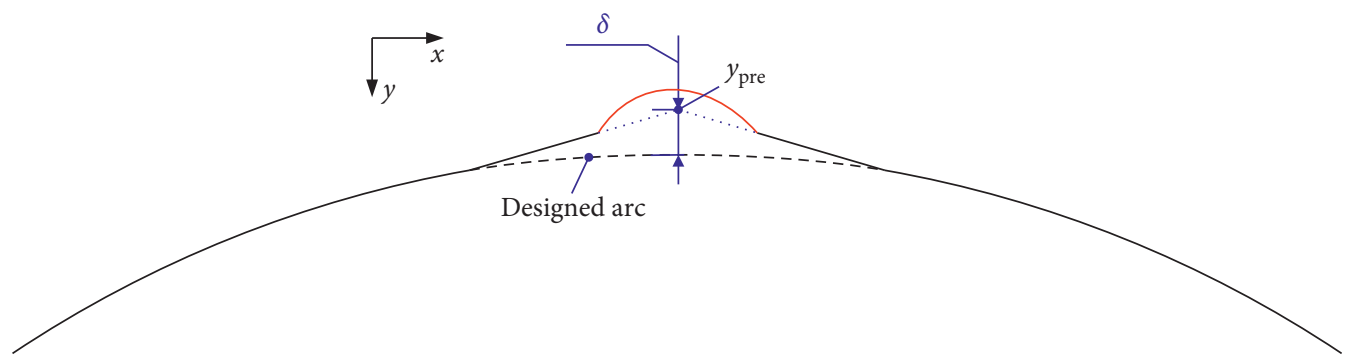

FIGURE 3: Longitudinal weld peaking estimation based on the standard.

$$
g(x)=\left(k+\mathrm{a}^{T} \lambda\right) x+m
$$

The prior conditions of the parameter are as follows: $k \sim N\left(0, \sigma^{2}\right), m \sim N\left(0, \sigma^{2}\right)$.

\section{Experiments and Results}

4.1. Experiment on the Girth Weld of the Pressure Pipe after Welding. To verify the feasibility of the PBLR method, the currently popular deep learning algorithms for sequence prediction, the LSTM algorithm, and the traditional leastsquares linear fitting linear regression (LR) algorithm were used to predict the coordinate positions of the contact points of the joints on both sides of the girth weld.

The LSTM network is a neural network used to process sequence data. Moreover, unlike general neural networks, it can process data with sequence changes. We considered the point set $\mathrm{M}_{\text {left }}$ of the base metal area of the left weld contour as an example and cut $M_{\text {left }}$ into an input sequence $I^{1}=\left\{M_{1}(x, y), \cdots, M_{n}(x, y)\right\}, \ldots, \quad$ and $\quad I^{\text {left }-n-m+1}=$ 


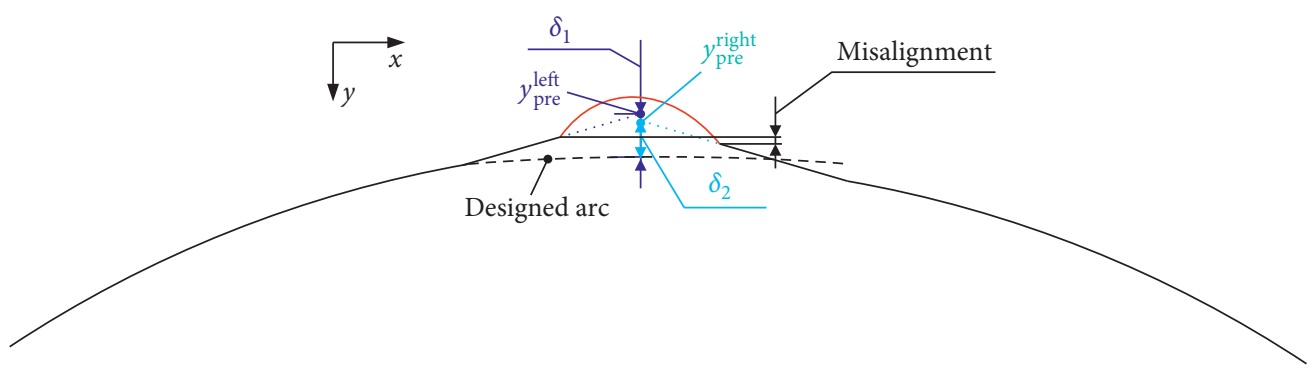

FIGURE 4: Longitudinal weld peaking estimation with misalignment.

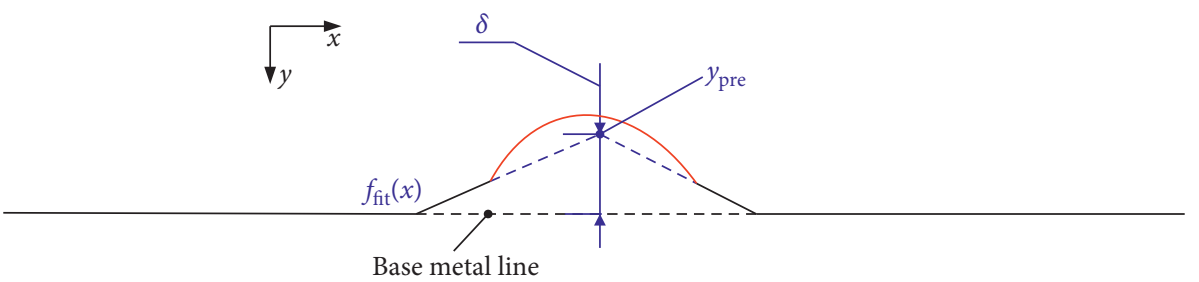

Figure 5: Girth weld peaking estimation based on the standard.

$\left\{M_{\text {left }-n-m+1}(x, y), \cdots, M_{\text {left } n-m}(x, y)\right\}$, and an output sequence $I^{m+1}=\left\{M_{m+1}(x, y), \cdots, M_{m+n}(x, y)\right\}, \ldots$, and $I^{\text {left }-n+1}=\left\{M_{\text {left }-n+1}(x, y), \cdots, M_{\text {left }}(x, y)\right\}$ with a sequence of length $n$ at equal intervals, where $m$ is the predicted length. The previous sequence content $I^{1}$ predicted the result of the subsequent $I^{m+1}$; thus, the training set of the LSTM network was related to the selected sequence length $n$ and the predicted length $m$. If the sequence lengths $n$ and $m$ were too large to cause the LSTM training set to be small enough, it resulted in a poor prediction ability of the LSTM network. The common forward calculation method of the LSTM network is expressed as follows:

$$
\begin{aligned}
i_{i} & =\sigma\left(W_{x i} I_{i}+W_{h i} h_{i-1}+W_{c i} c_{i-1}+b_{i}\right), \\
c_{i} & =f_{i} c_{i-1}+i_{i} \tanh \left(W_{x c} I_{i}+W_{h c} h_{i-1}+b_{c}\right), \\
f_{i} & =\sigma\left(W_{x f} I_{i}+W_{h f} h_{i-1}+W_{c f} c_{i-1}+b_{f}\right), \\
o_{i} & =\sigma\left(W_{x o} I_{i}+W_{h o} h_{i-1}+W_{c o} c_{i}+b_{o}\right), \\
h_{i} & =o_{i} \tanh \left(o_{i}\right)
\end{aligned}
$$

where $i, f, c$, and $o$ are the input gate, forget gate, cell state, and output gate, respectively. $W$ and $b$ are the corresponding weight coefficient matrix and bias term, respectively. $\sigma$ and tanh are the sigmoid and hyperbolic tangent activation functions, respectively. The LSTM calculation process can be divided into 4 steps: (1) calculating the output value of the LSTM cell based on equation (6), (2) calculating the error item of each LSTM cell in reverse, including two backward propagation directions based on the time and network level, (3) calculating the gradient of each weight according to the corresponding error term, and (4) applying a gradient-based optimization algorithm to update the weights. The training LSTM hyperparameters used in this article were set as follows: the number of the training steps of the LSTM method was 10,000 , the sequence length $n$ was 20 , and the number of hidden layer nodes was 8 .
The standard linear regression model of the LR method was set as $y=a x+b$, and the base metal profile data and regression analysis on both sides were performed by the least square method.

The number of the selected change points of the important parameters in the PBLR model affects the complexity of the regression model. Here, $1 / 10$ of the training sample was selected. The changing trend of the weld base metal contour was relatively single, and the equal interval selection method could be used in the selection of $c p_{i}$. The regression prior conditions of the PBLR model parameters were $\lambda \sim \operatorname{Laplace}(0,0.8), k \sim N(0,1)$, and $m \sim N(0,1)$. Using Stan language to establish the PBLR model, the iterative condition was set as 10,000 iterative steps, and the optimization method was Newton's iterative method. The PBLR model was used to predict the coordinate positions of the contact points of the LSTM and LR methods on both sides of the girth weld based on the profile data $M_{\text {left }}$ and $M_{\text {right }}$ of the base metal on both sides. The surface profiles of the different positions of the girth weld with diameters of $120 \mathrm{~mm}, 130 \mathrm{~mm}$, and $140 \mathrm{~mm}$ were collected. The base metal on the left side of the cylinder demonstrated an obvious downward depression at the position close to the weld. Figure 7 shows the prediction effect.

From the above comparison chart, it can be seen that the LSTM method has a large deviation due to less training set in the case of a large weld width, as shown in Figures 7(a), 7(c), and $7(d)$, from the data of the base metal on the right side. In the case of abundant training sets, the effect was close to that of the PBLR method, as shown in Figures 7(b) and 7(d), and was predicted from the left base metal data. On the other hand, the LR method has better prediction effects in the case of no obvious change in the base metal contour, as shown in Figures $7(\mathrm{c})-7(\mathrm{f})$, on the right but has poor prediction effects in the case of a considerable change in the base metal contour, i.e., bending of the base metal, as shown in Figures 7(a)-7(f), on the left. Compared with the LSTM and 


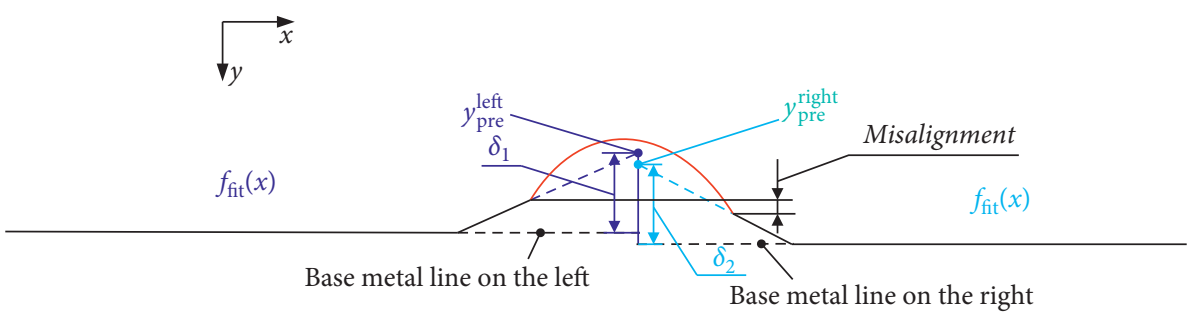

FIgURE 6: Girth weld peaking estimation with misalignment.

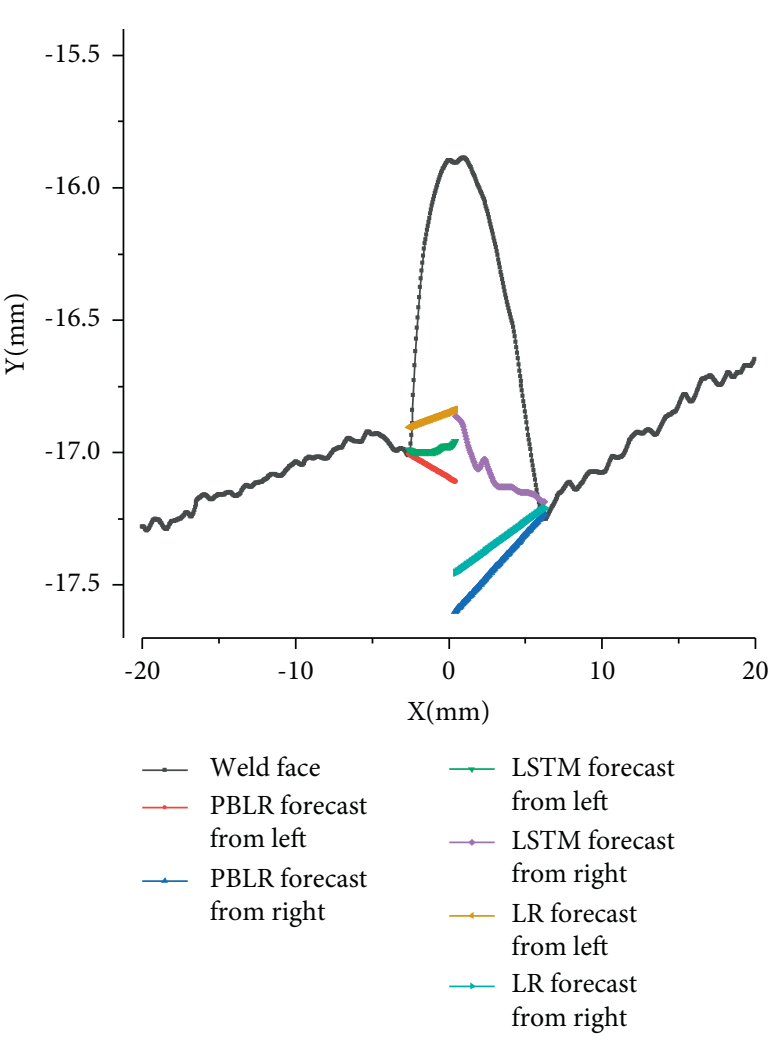

(a)

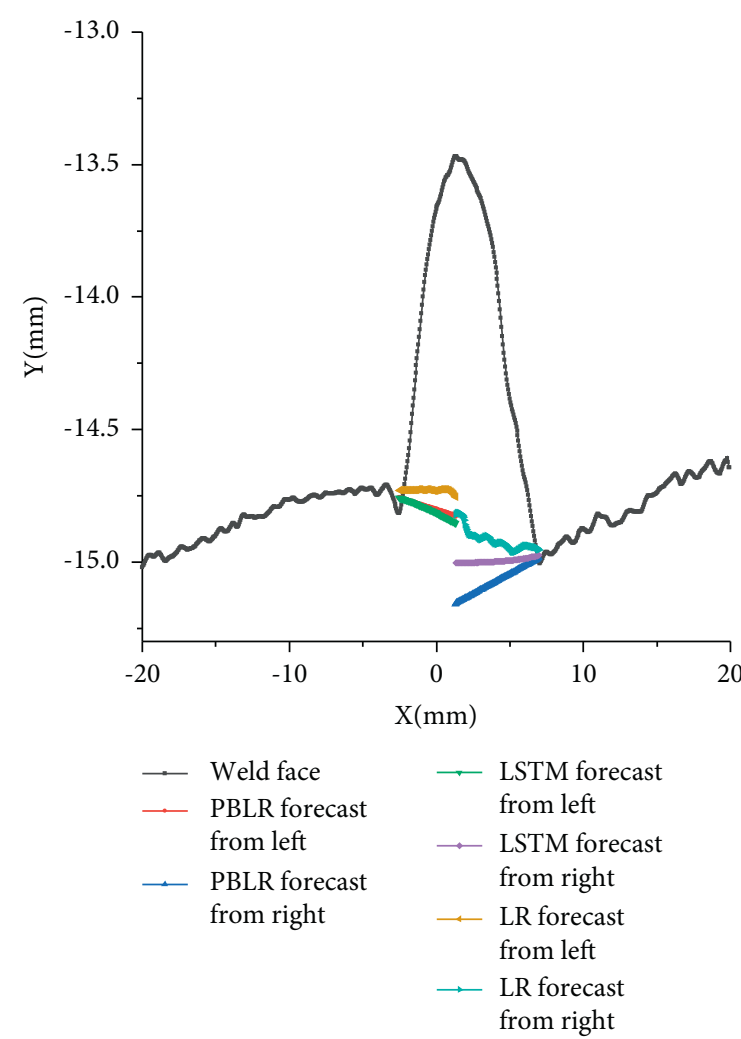

(b)

Figure 7: Continued. 


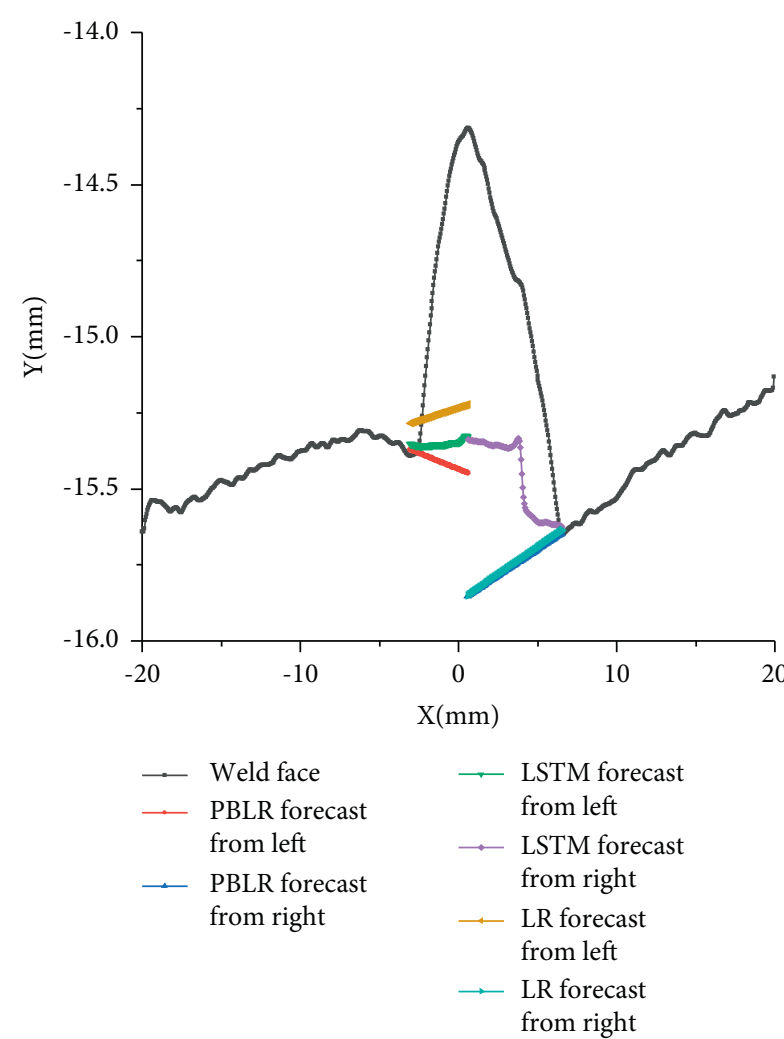

(c)

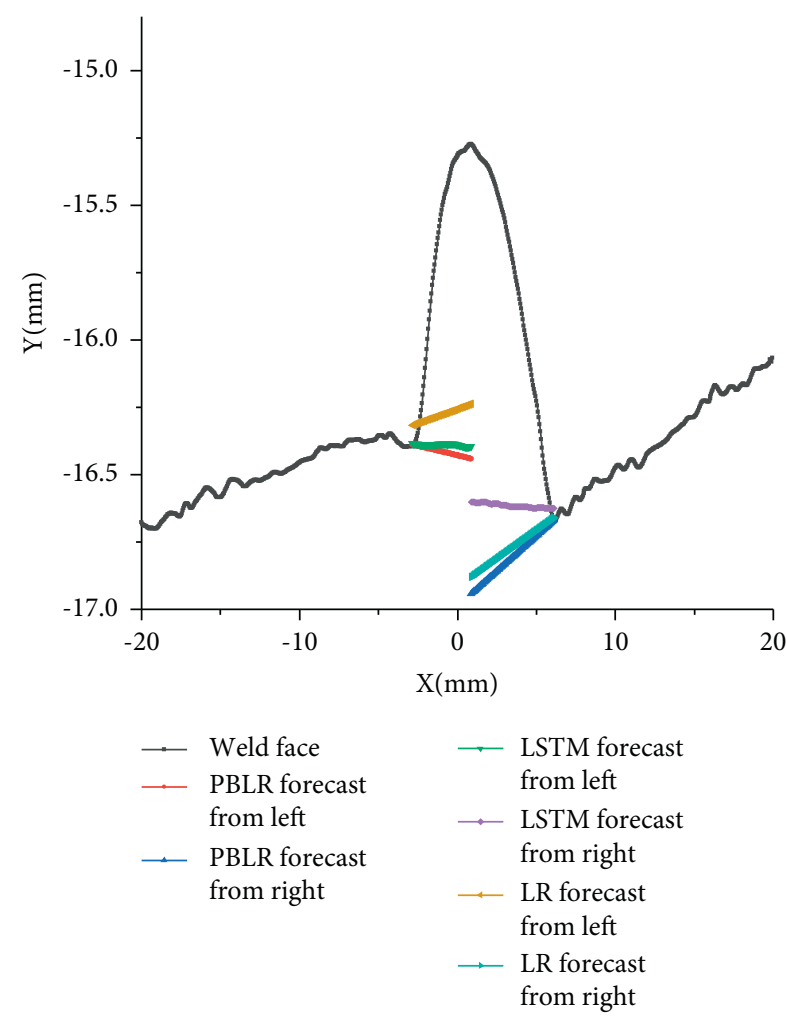

(e)

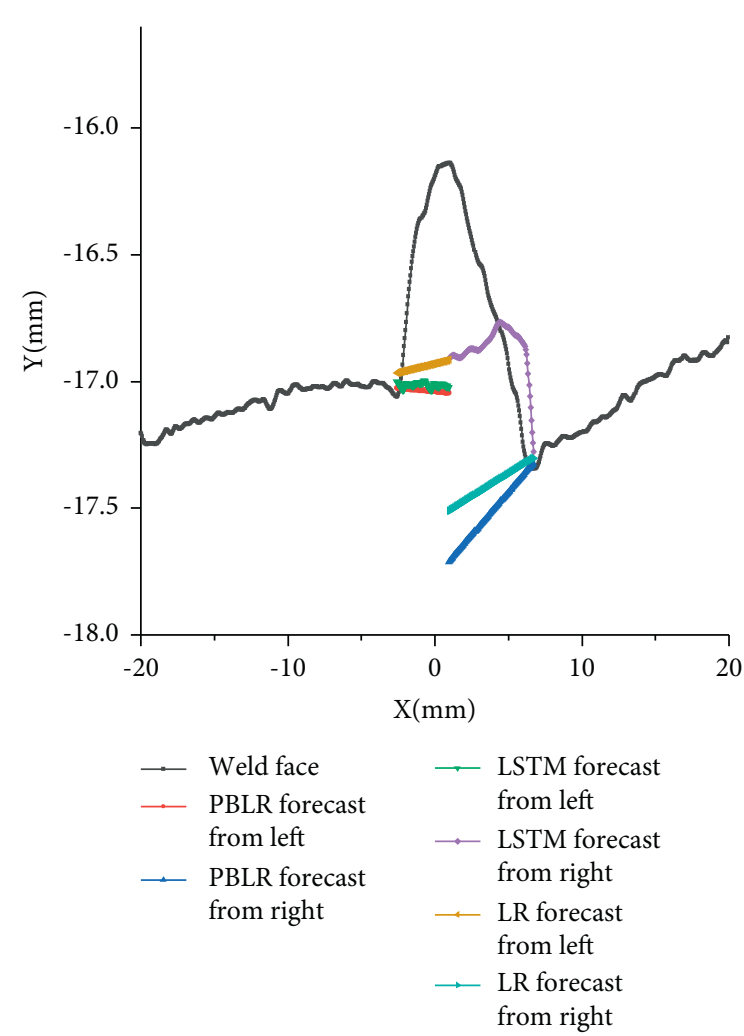

(d)

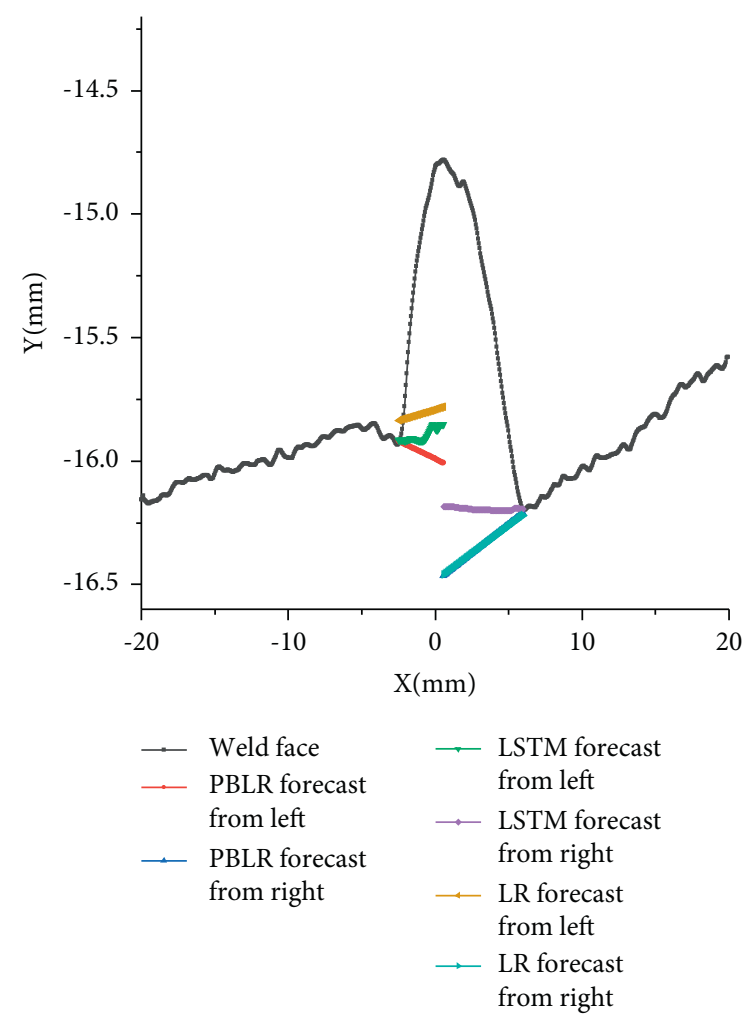

(f)

FIgURE 7: Girth weld peaking estimation based on the standard. (a, b) a pressure pipe with a diameter of $120 \mathrm{~mm}$; (c, d) a pressure pipe with a diameter of $130 \mathrm{~mm}$; (e, f) a pressure pipe with a diameter of $140 \mathrm{~mm}$. 


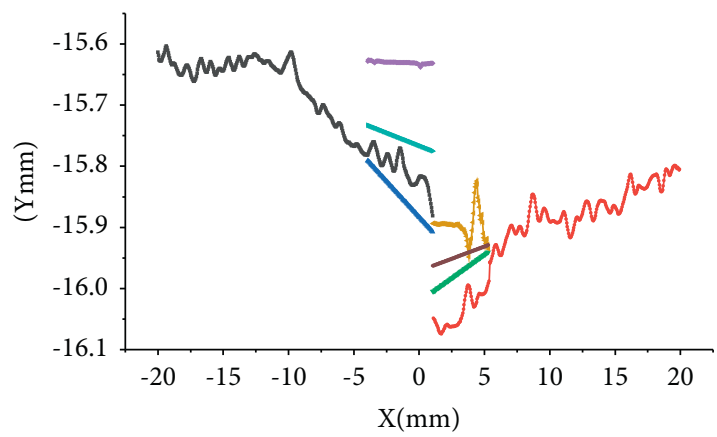

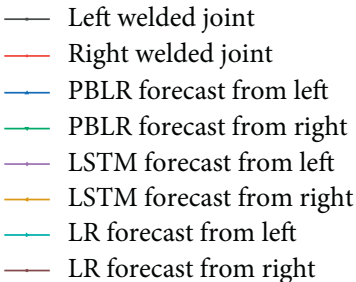

(a)

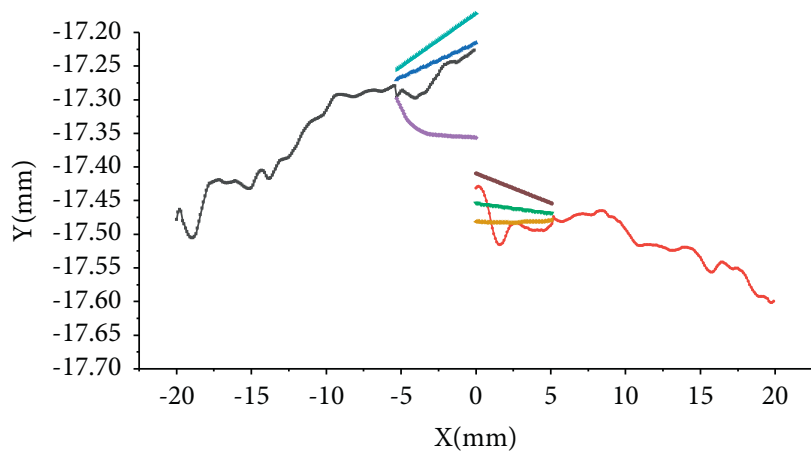

— PBLR forecast from left —- Welded joint left

— PBLR forecast from right _— Welded joint right

— LSTM forecast from left

—_ LSTM forecast from right

$\rightarrow$ LR forecast from left

— LR forecast from right

FIgure 8: Girth weld peaking estimation with misalignment. (a) Pressure pipe with a diameter of $170 \mathrm{~mm}$ and (b) pressure pipe with a diameter of $200 \mathrm{~mm}$.

TABLE 1: Estimated results of the three methods PBLR, LSTM, and LR (unit: $\mathrm{mm}$ ).

\begin{tabular}{lccc}
\hline Estimation error (mean absolute value) & PBLR & LSTM & LR \\
\hline From left side & 0.043 & 0.314 & 0.281 \\
From right side & 0.054 & 0.368 & 0.394 \\
Peaking theoretical measurement error & 0.061 & 0.426 & 0.439 \\
\hline
\end{tabular}

TABLE 2: Estimated deviation of the PBLR, LSTM, and LR methods at 5 different joints on the left and right sides of the girth weld with the diameters of $170 \mathrm{~mm}$ and $200 \mathrm{~mm}$ (unit: $\mathrm{mm}$ ).

\begin{tabular}{|c|c|c|c|c|c|c|c|}
\hline \multirow{2}{*}{\multicolumn{2}{|c|}{ Position (mm) }} & \multicolumn{2}{|c|}{ PBLR } & \multicolumn{2}{|c|}{ LSTM } & \multicolumn{2}{|c|}{ LR } \\
\hline & & Left & Right & Left & Right & Left & Right \\
\hline \multirow{5}{*}{170} & 1 & 0.044 & 0.077 & 0.267 & 0.422 & 0.209 & 0.106 \\
\hline & 2 & 0.030 & 0.045 & 0.642 & 0.575 & 0.096 & 0.188 \\
\hline & 3 & 0.038 & 0.069 & 0.359 & 0.158 & 0.245 & 0.604 \\
\hline & 4 & 0.031 & 0.056 & 0.148 & 0.179 & 0.186 & 0.582 \\
\hline & 5 & 0.068 & 0.073 & 0.276 & 0.275 & 0.133 & 0.542 \\
\hline \multirow{5}{*}{200} & 1 & 0.048 & 0.042 & 0.641 & 0.383 & 0.197 & 0.297 \\
\hline & 2 & 0.030 & 0.047 & 0.110 & 0.169 & 0.121 & 0.345 \\
\hline & 3 & 0.056 & 0.050 & 0.196 & 0.348 & 0.411 & 0.330 \\
\hline & 4 & 0.034 & 0.040 & 0.193 & 0.736 & 0.313 & 0.289 \\
\hline & 5 & 0.047 & 0.051 & 0.540 & 0.280 & 0.433 & 0.640 \\
\hline
\end{tabular}

LR methods, the PBLR method was more stable in the prediction effect and could adapt to the mutation of the left side depression of the base metal.

4.2. Experiment on the Girth Weld Joint before Welding. The surface profiles of 30 different positions of the girth welded joints of a rolled cylinder with the diameters of $170 \mathrm{~mm}$ and $200 \mathrm{~mm}$ were collected, and the contour points of the base metal in the welding area were predicted using the three aforementioned methods. The prediction effect is shown in Figure 8. The measurement error of the girth weld joint peaking based on the PBLR contour prediction method mainly comes from the installation error of the measuring device, the estimation error, and the base metal linear fitting error. Ideally, the installation and estimation errors of the measuring device can be regarded as 0 . Therefore, the theoretical measurement error based on the PBLR girth weld joint peaking can be determined as the estimation error. Table 1 shows the mean absolute value of the estimated 
deviation of the three methods, PBLR, LSTM, and LR, at 30 different joints on the left and right sides of the girth weld and the mean absolute value of the theoretical error of the peaking measurement. Table 2 shows the estimated deviation of the PBLR, LSTM, and LR methods at 10 different joints on the left and right sides of the girth weld with diameters of $170 \mathrm{~mm}$ and $200 \mathrm{~mm}$, the deviation between the PBLR predicted and actual contours was smaller than that by the LSTM and LR methods, and the theoretical mean absolute error of the peaking measured by this method could attain $0.06 \mathrm{~mm}$, which meets the detection accuracy requirements of the relevant detection units.

4.3. Experiment on the Longitudinal Weld of the Pressure Pipe after Welding. Both sides of the base metal of the longitudinal weld of the pressure pipeline were elliptical, similar to the abovementioned girth weld comparative experimental design. The PLR, LSTM, and the least square-based ellipse fitting (EF) methods were used.

The EF method considers the point set of the base metal area on both sides of the weld as $M=\left\{M_{1}(x, y), \cdots\right.$, $\left.M_{k}(x, y)\right\}$, and the target regression curve can establish a quadratic curve equation with the regression parameter $U=\left(u_{1}, u_{2}, u_{3}, u_{4}, u_{5}\right)$, namely,

$$
f(x, y)=u_{1} x^{2}+u_{2} x y+u_{3} y^{2}+u_{4} x+u_{5} y+1 .
$$

Based on the solving principle of the least-squares method, the quadratic curve fitting is transformed into a quadratic curve. The sum of the squares of the algebraic distances at all positions of the point set is $\sum_{i=1}^{k} f\left(x_{i}, y_{i}\right)=0$, and the least-squares method of the quadratic curve is solved with $\partial f / \partial u_{i}=0, i=1, \cdots, 5$. However, under normal circumstances, the diameter of the pressure pipe cylinder is large, and the solution is more complicated. The base material point set coordinate $M_{i}(x, y)$ can be normalized, that is, using the coordinate transformation $\tilde{M}_{i}=T M_{i}$, where $T$ is given by

$$
T=\left[\begin{array}{ccc}
s & 0 & -s t_{x} \\
0 & s & -s t_{y} \\
0 & 0 & 1
\end{array}\right],
$$

where $s$ is the zoom factor and $\left(t_{y}, t_{y}\right)$ is the translation vector. The relationship between the two is given by the following equation:

$$
\begin{aligned}
s & =\frac{\sqrt{2}}{\sum_{i=1}^{k} \sqrt{\left(x_{i}-t_{x}\right)^{2}+\left(y_{i}-t_{y}\right)^{2}}} \\
t_{x} & =\frac{\sum x_{i}}{k} \\
t_{y} & =\frac{\sum y_{i}}{k}
\end{aligned}
$$

To distinguish the fitted quadratic curve from a hyperbola, parabola, etc., the following constraints can be added to ensure that it becomes a circle or an ellipse, and the diameter of the cylindrical part to be tested is $R_{\mathrm{stand}}$. Therefore,

$$
\text { s.t. }\left\{\begin{array}{l}
u_{2}^{2}-4 u_{1} u_{3}<0, \\
\left(u_{1}+u_{3}\right)\left(4 u_{1} u_{3}-u_{1} u_{5}^{2}-u_{2}^{2}+u_{2} u_{4} u_{5}-u_{4}^{2} u_{3}\right)<0 \\
\sqrt{\frac{2 u_{1} x_{c}^{2}+2 u_{3} y_{c}^{2}+u_{2} x_{c} y_{c}-1}{u_{1}+u_{3}+\sqrt{\left(u_{1}-u_{3}\right)^{2}+u_{2}^{2}}}}=\alpha R_{\mathrm{stand}} \\
\sqrt{\frac{2 u_{1} x_{c}^{2}+2 u_{3} y_{c}^{2}+u_{2} x_{c} y_{c}-1}{u_{1}+u_{3}-\sqrt{\left(u_{1}-u_{3}\right)^{2}+u_{2}^{2}}}}=R_{\mathrm{stand}}
\end{array}\right.
$$

where $\alpha$ is the ellipsification factor.

The parameter selection method in the PLR model is the same as that of the PBLR model used in the girth weld. The prior conditions for the regression of the PLR model parameters are $\lambda \sim \operatorname{Laplace}(0,0.8), k \sim N(0,1), b \sim N(0,1)$, and $\beta \sim N(0,1)$. The Stan language is used to establish the PLR model, and the set iterative condition is 10,000 steps. The optimization method is Newton's iteration method. Figure 9 shows the prediction results of the three estimation methods with the design diameters of $155 \mathrm{~mm}$ and $550 \mathrm{~mm}$.

It can be seen from Figure 9 that the prediction effect of the LSTM method under the longitudinal weld is consistent with that of the girth weld. Fewer training samples may lead to larger prediction deviations, as shown in Figure 9(a), on the right side of the base metal data prediction effect. From the perspective of the forecast trend, the overall forecast trend of LSTM does not conform to the outline. The trend is shown in Figures 9(b) and 9(c). In the pressure pipeline with a small misalignment in Figures 9(a)-9(c), the EF method predicts a better contour effect, but when there exists a large misalignment, the predicted direction has a large deviation, as shown in Figure 10(d). In contrast, the overall prediction result of the PLR method is more accurate and can better predict the trend of the base metal profile even in the presence of large misalignment.

4.4. Experiment on the Longitudinal Weld Joint before Welding. The surface profiles of 30 different joints of the longitudinal welded joints of the rolled cylinder with diameters of $255 \mathrm{~mm}$ and $600 \mathrm{~mm}$ were collected. The contour points of the base metal in the welding area were predicted using the above three methods. Figure 10 shows the prediction effect. Table 3 shows the PLR at 30 different joint positions. The two models LSTM and EF estimate the mean values of the absolute deviation and theoretical error in the longitudinal weld joints at the left and right sides of the weld, respectively. Table 4 shows the estimated deviation of the PLR, LSTM, and EF methods at 10 different joints on the left and right sides of the longitudinal weld with the diameters of $170 \mathrm{~mm}$ and $200 \mathrm{~mm}$. It can be observed from the results 


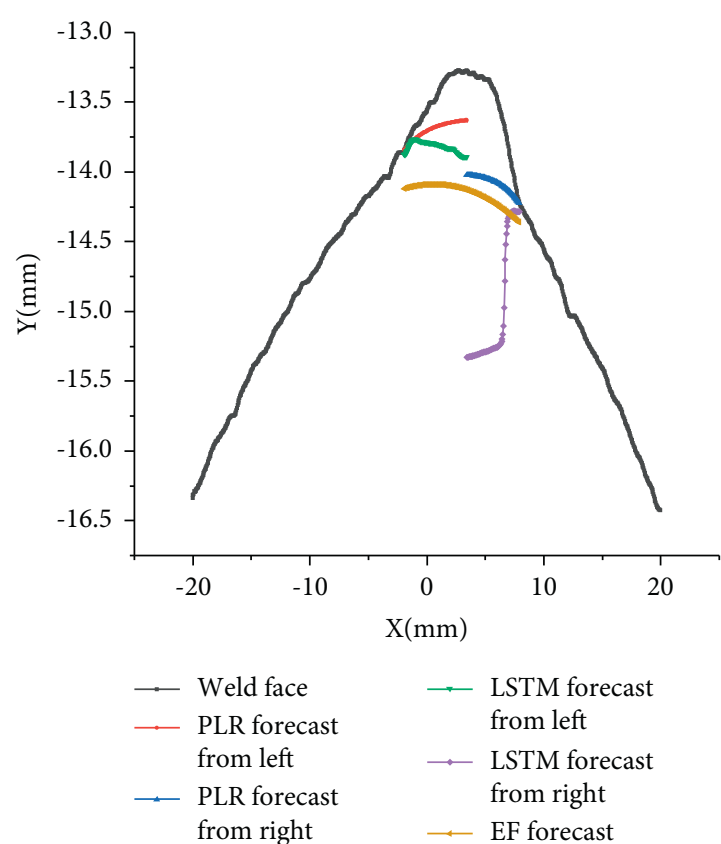

(a)
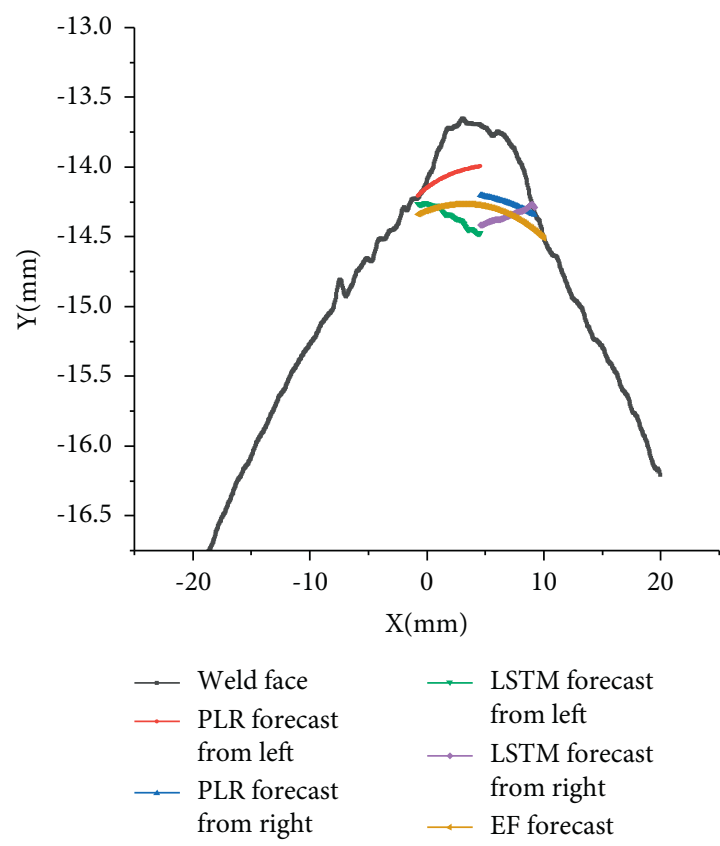

(c)

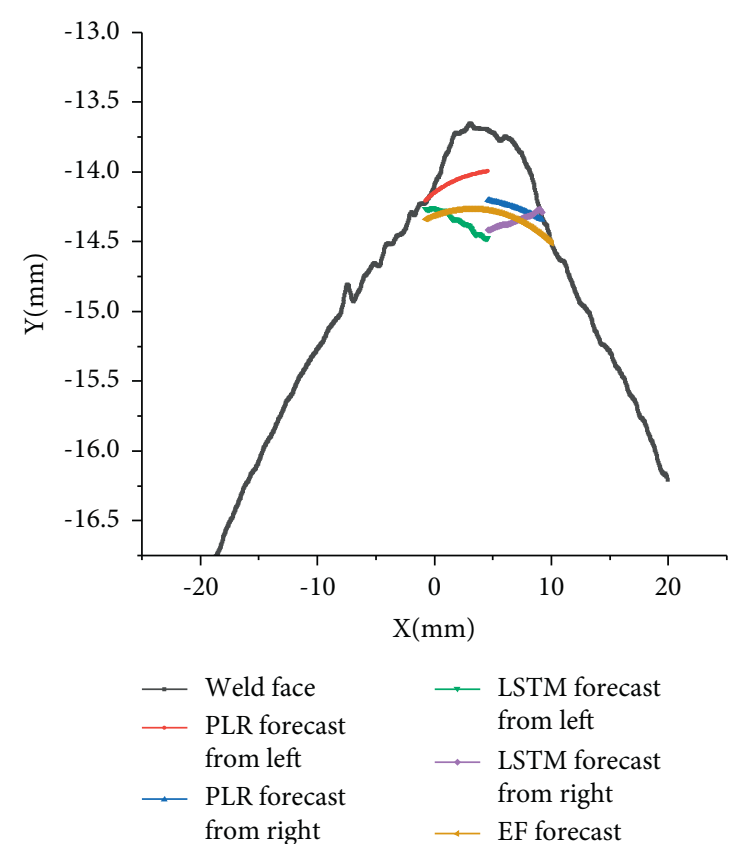

(b)

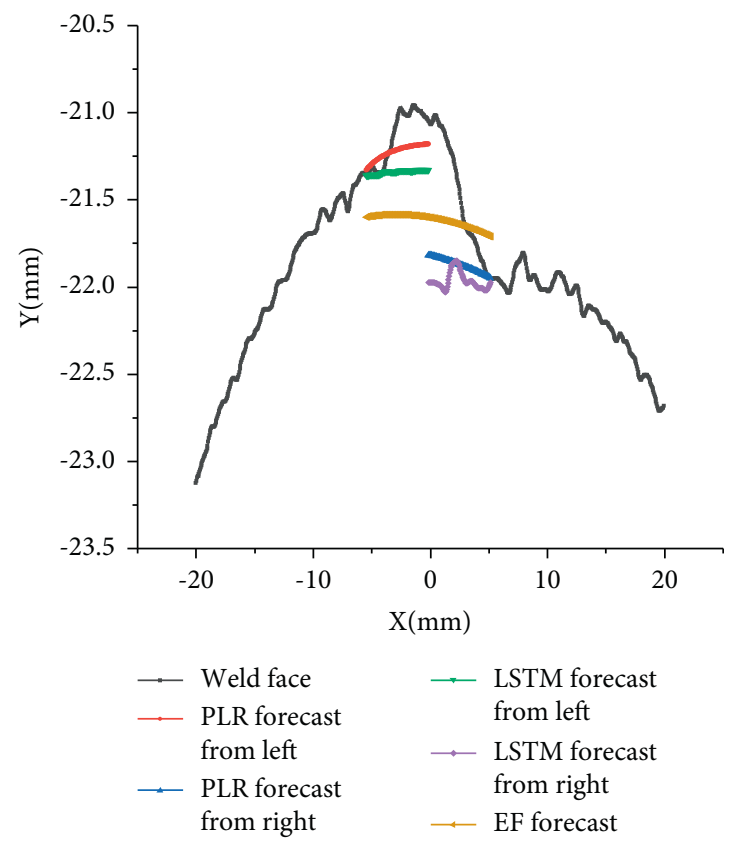

(d)

FIGURe 9: Girth weld peaking estimation based on the standard. (a-c) Pressure pipe with a diameter of $155 \mathrm{~mm}$. (d) Pressure pipe with a diameter of $550 \mathrm{~mm}$.

that the mean value of the absolute error of the deviation between the predicted and actual contours is smaller when using the PLR, and the theoretical mean absolute error of the peaking measured by this method can attain $0.07 \mathrm{~mm}$, which meets the requirements of the detection accuracy of the relevant detection units. 


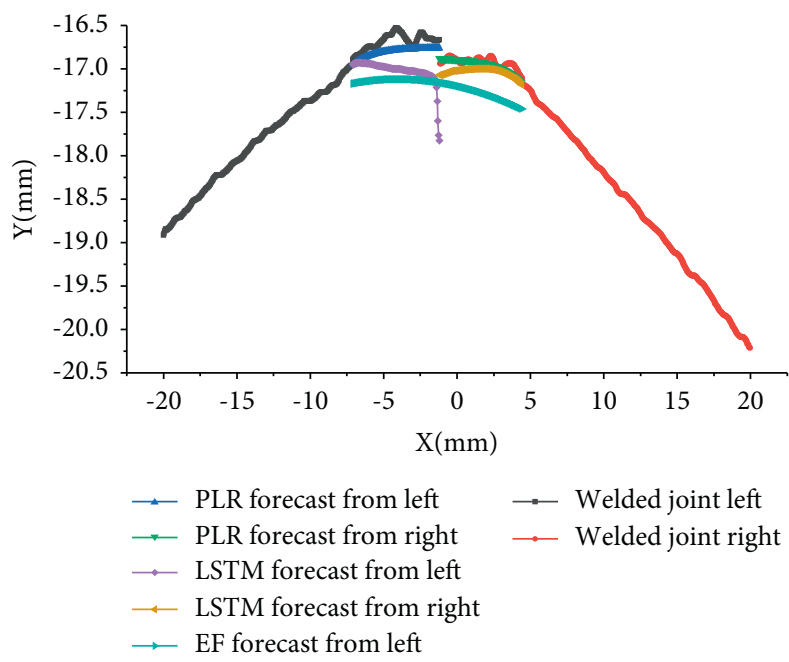

(a)

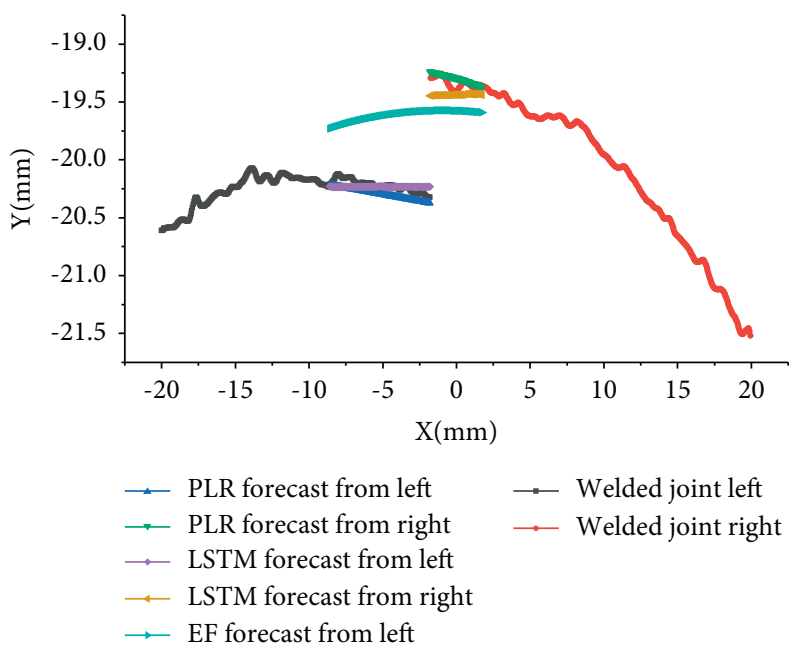

(b)

Figure 10: The girth weld peaking estimation with misalignment. (a) Pressure pipe with a diameter of $255 \mathrm{~mm}$. (b) Pressure pipe with a diameter of $600 \mathrm{~mm}$.

TABLE 3: Estimated results of the three methods PLR, LSTM, and EF (unit: mm).

\begin{tabular}{lccc}
\hline Estimation error (mean absolute value) & PLR & LSTM & EF \\
\hline From left side & 0.050 & 0.334 & 0.331 \\
From right side & 0.062 & 0.483 & 0.437 \\
Peaking theoretical measurement error & 0.072 & 0.546 & 0.459 \\
\hline
\end{tabular}

TABLE 4: Estimated deviation of the PLR, LSTM, and EF methods at 10 different joints on the left and right sides of the longitudinal weld with the diameters of $170 \mathrm{~mm}$ and $200 \mathrm{~mm}$ (unit: $\mathrm{mm}$ ).

\begin{tabular}{|c|c|c|c|c|c|c|c|}
\hline \multirow{2}{*}{\multicolumn{2}{|c|}{ Position $(\mathrm{mm})$}} & \multicolumn{2}{|c|}{ PLR } & \multicolumn{2}{|c|}{ LSTM } & \multicolumn{2}{|c|}{$\mathrm{EF}$} \\
\hline & & Left & Right & Left & Right & Left & Right \\
\hline \multirow{5}{*}{255} & 1 & 0.047 & 0.056 & 0.142 & 0.205 & 0.337 & 0.795 \\
\hline & 2 & 0.036 & 0.054 & 0.102 & 0.295 & 0.283 & 0.687 \\
\hline & 3 & 0.049 & 0.071 & 0.537 & 0.731 & 0.242 & 0.221 \\
\hline & 4 & 0.063 & 0.062 & 0.199 & 0.281 & 0.253 & 0.182 \\
\hline & 5 & 0.057 & 0.069 & 0.348 & 0.321 & 0.248 & 0.145 \\
\hline \multirow{5}{*}{600} & 1 & 0.042 & 0.064 & 0.566 & 0.480 & 0.356 & 0.404 \\
\hline & 2 & 0.052 & 0.075 & 1.155 & 0.779 & 0.678 & 0.468 \\
\hline & 3 & 0.062 & 0.064 & 0.219 & 0.477 & 0.188 & 0.488 \\
\hline & 4 & 0.033 & 0.056 & 0.601 & 1.189 & 0.273 & 0.397 \\
\hline & 5 & 0.052 & 0.078 & 0.112 & 0.395 & 0.111 & 0.697 \\
\hline
\end{tabular}

\section{Conclusions}

Aiming to solve the problem that the peaking measurement method defined by the standard cannot be effectively used in the appearance detection of pressure vessel welding without polished reinforcement and misalignment, we propose a regression-based estimation method. In the proposed method, we first use a laser profile sensor to obtain a set of weld surface contour points, and then based on the contour characteristics of the base metal of the girth weld and the longitudinal weld, we introduce discontinuous point information on the basis of logistic regression and Bayesian linear regression models, divide a single regression model into multiple interrelated piecewise regression models, and modify the model prediction trend and bias value in each segment of the model. Then, we collected a variety of pressure pipeline weld area profile data before and after welding and compared our proposed PLR and PBLR methods with the existing LSTM method, linear regression and elliptic regression methods in terms of prediction effects. The results showed that the accuracies of the PBLR and PLR methods were better, and the theoretical mean absolute error of the peaking measured at different positions could attain approximately $0.8 \mathrm{~mm}$. In the future, we will design an 
experimental device with higher positioning accuracy to achieve high-precision detection of the weld peaking of the pressure pipeline.

\section{Data Availability}

All data used to support the findings of this study are included within the article.

\section{Conflicts of Interest}

The authors declare that there are no conflicts of interest.

\section{Authors' Contributions}

P.L., G.L., and N.Y. conceived the study concept; P.L. and N.Y. performed the experiments; P.L. and G.L. carried out the system model; and P.L. wrote the manuscript.

\section{Acknowledgments}

This research was funded by the Science and Technology Plan Project of the State Administration for Market Regulation, grant no. 2019MK143.

\section{References}

[1] G. E. Cook, R. J. Barnett, K. Andersen, J. F. Springfield, and A. M. Strauss, "Automated visual inspection and interpretation system for weld quality evaluation," in Proceedings of the Industry Applications Conference, Orlando, FL, USA, October 1995.

[2] J. Zapata, R. Vilar, and R. Ruiz, "Performance evaluation of an automatic inspection system of weld defects in radiographic images based on neuro-classifiers," Expert Systems with Applications, vol. 38, no. 7, pp. 8812-8824, 2011.

[3] G. Baylac, I. Roberts, S. Maddox, and E. Zeelenberg, "Non-destructive testing and inspection of unfired pressure vessels subject to cyclic loads in EN 13445-5," in Proceedings of the Asme Pressure Vessels \& Piping Conference, July 2005.

[4] A. Schwarz, Method and Sensor Mount for Measuring Seam Peaking in Pipes by Means of Ultrasonic Inspection, Google Patents, 2016.

[5] S. Michael, D. Jonathan, and E. Phillip, Remaining Life Sensitivity to Longitudinal Weld Seam Peaking in High-Temperature Low Chrome Piping, 2016.

[6] G. Buchheim, D. Osage, R. Brown, and J. Dobis, "Failure investigation of a low chrome long-seam weld in a high-temperature refinery piping system," Journal of Pressure Vessel Technology, vol. 117, no. 3, pp. 227-237, 1995.

[7] P. E. Prueter, Techniques for Simulating Creep Damage Evolution at Welds with Emphasis on Evaluating Longitudinal Seam Peaking in High-Temperature Piping Systems, Pressure Technology, American Society of Mechanical Engineers (ASME), New York, NY, USA, 2018.

[8] G. Casalino, M. Moradi, M. K. Moghadam, A. Khorram, and P. Perulli, "Experimental and Numerical study of AISI 4130 steel surface hardening by Pulsed Nd:YAG laser," Materials, vol. 12, no. 19, p. 3136, 2019.

[9] J. Patel, G. Chauhan, D. Raghuwanshi, and A. Mohanan, The Automated System for Line Pipe's Dimensions Measurement, ASME India Oil and Gas Pipeline Conference, American
Society of Mechanical Engineers (ASME), New York, NY, USA, 2017.

[10] H. He, H. Zhao, W. Niu, and P. Wang, "A method to measure welding deformation of plate by three dimensional laser scanner," vol. 32, no. 12, pp. 9-12, 2011.

[11] Fumin, Zhang, and Xinghua, "An automated inner dimensional measurement system based on a laser displacement sensor for long-stepped pipes," Sensor, vol. 12, no. 5, pp. 5824-5834, 2012.

[12] J. Fan, F. Jing, L. Yang, T. Long, and M. Tan, “A precise initial weld point guiding method of micro-gap weld based on structured light vision sensor," Sensor, vol. 19, p. 1, 2018.

[13] L. Yang, E. Li, T. Long, J. Fan, and Z. Liang, "A Novel 3-D Path extraction method for arc welding Robot based on Stereo structured light sensor," IEEE Sensors Journal, vol. 19, no. 2, pp. 763-773, 2019.

[14] F. A. Gers, N. N. Schraudolph, and J. Schmidhuber, "Learning precise timing with lstm recurrent networks," Journal of Machine Learning Research, vol. 3, no. 1, pp. 115-143, 2003.

[15] K. Greff, R. K. Srivastava, J. Koutník, and B. R. Steunebrink, "LSTM: A Search Space Odyssey," vol. 28, no. 10, pp. 2222-2232, 2016, https://arxiv.org/abs/1503.04069.

[16] A. Aminzadeh, A. Parvizi, and M. Moradi, "Multi-objective topology optimization of deep drawing dissimilar tailor laser welded blanks," Experimental and Finite Element Investigation, vol. 125, 2020.

[17] G. Ye, J. Guo, Z. Sun, C. Li, S. Zhong, and C.-I. Manufacturing, "Weld bead recognition using laser vision with model-based classification," Robotics and Computer-Integrated Manufacturing, vol. 52, pp. 9-16, 2018.

[18] K. Zhang, M. Yan, T. Huang, J. Zheng, and Z. Li, "3D reconstruction of complex spatial weld seam for autonomous welding by laser structured light scanning," Journal of Manufacturing Processes, vol. 39, pp. 200-207, 2019.

[19] T. Liu, J. Bao, J. Wang, and Y. J. S. Zhang, "A Hybrid CNN-LSTM algorithm for online defect recognition of $\mathrm{CO} 2$ welding," vol. 18, no. 12, 2018.

[20] Y. Wang, C. Zhang, J. Lu, L. Bai, Z. Zhao, and J. Han, "Weld reinforcement analysis based on long-term prediction of molten pool image in Additive manufacturing," IEEE Access, vol. 8, pp. 69908-69918, 2020.

[21] R. Hartl, B. Praehofer, and M. Zaeh, "Prediction of the surface quality of friction stir welds by the analysis of process data using Artificial Neural Networks," Proceedings of the Institution of Mechanical Engineers - Part L: Journal of Materials: Design and Applications, vol. 234, no. 5, pp. 732-751, 2020. 